\title{
Multimode model for an atomic Bose-Einstein condensate in a ring-shaped optical lattice
}

\author{
D. M. Jezek and H. M. Cataldo \\ IFIBA-CONICET Pabellón 1, Ciudad Universitaria, 1428 Buenos Aires, Argentina \\ (Received 10 October 2012; revised manuscript received 5 June 2013; published 29 July 2013)
}

\begin{abstract}
We study the population dynamics of a ring-shaped optical lattice with a high number of particles per site and a low (less than ten) number of wells. Using a localized on-site basis defined in terms of stationary states, we were able to construct a multiple-mode model depending on relevant hopping and on-site energy parameters. We show that in the case of two wells, our model corresponds exactly to a recent improvement of the two-mode model. We derive a formula for the self-trapping period, which turns out to be chiefly ruled by the on-site interaction energy parameter. By comparing to time-dependent Gross-Pitaevskii simulations, we show that the multimode model results can be enhanced in a remarkable way over all the regimes by only renormalizing such a parameter. Finally, using a different approach which involves only the ground-state density, we derive an effective interaction energy parameter that turns out to be in accordance with the renormalized one.
\end{abstract}

DOI: 10.1103/PhysRevA.88.013636

PACS number(s): 03.75.Lm, 03.75.Hh, 03.75.Kk

\section{INTRODUCTION}

The two-mode model applied to double-well atomic Bose-Einstein condensates has been extensively studied in recent years [1-9]. Assuming that the order parameter can be described as a superposition of localized on-site wave functions with time-dependent coefficients, such a model predicts Josephson and self-trapping regimes [1,2], which have been experimentally observed by Albiez et al. [5].

The self-trapping (ST) phenomenon, which is also present in extended optical lattices [10], is a nonlinear effect where an initially highly populated (over a critical value) site remains with a larger number of particles than the remaining sites over the whole evolution. There is now active research on the self-trapping effect, which involves different types of systems, including mixtures of atomic species [11].

The dynamics of ring-shaped optical lattices with three [12] and four wells [13] has been previously investigated through multiple-mode (MM) models which utilized ad hoc values for hopping and on-site energy parameters. In the present article, instead, we will extract such parameters from a mean-field approach using localized on-site functions. We have shown in a previous work [14] that in a ring-shaped optical lattice, localized on-site [which we called Wannier-like (WL)] functions can be obtained in terms of stationary states of the Gross-Pitaevskii (GP) equation with different winding numbers. Here we will show that the above parameters yield the same type of corrections to the MM model for large filling factors as those obtained for the improved two-mode (TM) model for two-well systems [3].

We will derive an approximate formula for the self-trapping period in terms of the on-site interaction energy parameter. Using this formula and a single GP simulation result, a renormalizing on-site energy parameter that substantially improves the MM model can be obtained in what will be called the renormalized multiple-mode (RMM) model. Taking into account the density deformation during the time evolution [15], it has been shown in a recent work that for a double-well system an effective interaction energy parameter should be considered in the TM model to properly describe the exact dynamics [9]. Here we will adapt the same approach to our multiple-well system, which will allow us to obtain such an effective parameter only in terms of the ground-state density. Finally, we will show that both approaches give similar results.

This paper is organized as follows. In Sec. II we describe the system, and in Sec. III we outline the method for obtaining the WL functions, along with the properties required for building a reasonable multimode model dynamics. There we also define the model parameters in terms of the WL functions. In Sec. IV we specialize to the case of two wells, showing that our treatment through the WL functions turns out to be exactly the same as the latest two-mode model formulation [3]. Next, by means of the formula derived for the ST period, we show that the two-mode model can be enhanced in a remarkable way by only renormalizing the on-site interaction energy parameter. In Sec. V we develop the multiple-mode model, which generalizes our finding of the previous section. Finally, based on the method described in Ref. [9], in Sec. VI we derive an effective interaction energy parameter and compare it with the renormalized one. To conclude, a summary of our work is presented in Sec. VII.

\section{RING-SHAPED LATTICE AND CONDENSATE PARAMETERS}

We consider a Bose-Einstein condensate of rubidium atoms confined by an external trap $V_{\text {trap }}$, consisting of a superposition of a toroidal term $V_{\text {toro }}$ and a lattice potential $V_{\mathrm{L}}(x, y)$ formed by radial barriers. Similar to the trap utilized in recent experiments $[16,17]$, the toroidal trapping potential in cylindrical coordinates reads

$$
V_{\text {toro }}(r, z)=\frac{m}{2}\left[\omega_{r}^{2} r^{2}+\omega_{z}^{2} z^{2}\right]+V_{0} \exp \left(-2 r^{2} / \lambda_{0}^{2}\right)
$$

where $m$ is the atom mass and $\omega_{r}$ and $\omega_{z}$ denote the radial and axial frequencies, respectively. We have set $\omega_{z} \gg \omega_{r}$ to suppress excitation in the $z$ direction. In particular, we have chosen $\omega_{r} /(2 \pi)=7.8 \mathrm{~Hz}$ and $\omega_{z} /(2 \pi)=173 \mathrm{~Hz}$, while for the laser beam we have set $V_{0}=100 \hbar \omega_{r}$ and $\lambda_{0}=6 l_{r}$, with $l_{r}=\sqrt{\hbar /\left(m \omega_{r}\right)}$. On the other hand, the lattice potential is formed by $N_{c}$ Gaussian barriers located at equally spaced 
angular positions $\theta_{k}=2 \pi k / N_{c}$, where $-\left[\left[\left(N_{c}-1\right) / 2\right]\right] \leqslant$ $k \leqslant\left[\left[N_{c} / 2\right]\right]$ with [[-]] denoting the integer part,

$$
\begin{aligned}
V_{\mathrm{L}}(x, y)= & V_{b} \sum_{k=-\left[\left[\left(N_{c}-1\right) / 2\right]\right]}^{\left[\left[N_{c} / 2\right]\right]} \exp \left\{-\frac{\left[\cos \left(\theta_{k}\right) y-\sin \left(\theta_{k}\right) x\right]^{2}}{\lambda_{b}^{2}}\right\} \\
& \times \Theta\left[\sin \left(\theta_{k}\right) y+\cos \left(\theta_{k}\right) x\right],
\end{aligned}
$$

where $\Theta$ denotes the Heaviside function. For the numerical calculations we have fixed the width of the Gaussians to $\lambda_{b}=$ $0.5 l_{r}$ and the barrier height to $V_{b}=80 \hbar \omega_{r}$. In the mean-field approximation, the stationary states are solutions of the GP equation [18],

$$
\left[-\frac{\hbar^{2}}{2 m} \nabla^{2}+V_{\text {trap }}(\mathbf{r})+g N\left|\psi_{n}(\mathbf{r})\right|^{2}\right] \psi_{n}(\mathbf{r})=\mu \psi_{n}(\mathbf{r}),
$$

where $\psi_{n}(\mathbf{r})$ denotes a two-dimensional (2D) order parameter [19] normalized to unity with winding number $n$ [20]. The vorticity is numerically imprinted following the procedure described in Ref. [21]. $N$ and $\mu$ denote, respectively, the number of particles and the chemical potential $\left(N=10^{5}\right.$ will be assumed over all the numerical calculations). The effective 2D coupling constant $g=g_{3 D} \sqrt{m \omega_{z} / 2 \pi \hbar}$ is written in terms of the three-dimensional (3D) coupling constant between the atoms $g_{3 D}=4 \pi a \hbar^{2} / m$, where $a=98.98 a_{0}$ denotes the $s$-wave scattering length of ${ }^{87} \mathrm{Rb}$, with $a_{0}$ being the Bohr radius. Technical advances have been recently achieved to obtain experimentally this type of condensate in ring-shaped optical lattices with an arbitrary number of sites [22].

\section{LOCALIZED STATES AND HOPPING AND ON-SITE ENERGY PARAMETERS}

In this section we will summarize the results obtained in a previous work [14] that will be used to describe the present dynamics. We are interested in studying the Josephson and ST regimes. Such a dynamics takes place when the ground-state chemical potential becomes smaller than the minimum of the effective potential barrier dividing two lattice sites [20].

\section{A. Localized WL states}

The stationary states $\psi_{n}(r, \theta)$ are obtained as the numerical solutions of Eq. (3) [20]. Assuming large barrier heights [14], the winding number $n$ will be restricted to the values $-\left[\left[\left(N_{c}-1\right) / 2\right]\right] \leqslant n \leqslant\left[\left[N_{c} / 2\right]\right][20]$. We have seen in Ref. [14] that stationary states of different winding number must be orthogonal and that the definition for the WL functions,

$$
w_{k}(r, \theta)=\frac{1}{\sqrt{N_{c}}} \sum_{n} \psi_{n}(r, \theta) e^{-i n \theta_{k}},
$$

corresponds indeed to well-localized functions on each $k$ site. In addition, it has been shown in Ref. [14] that the above orthogonality implies that the set of $N_{c}$ WL functions (4) located at different $k$ sites must also form an orthonormal set. In Fig. 1 we have depicted the WL function density $w_{0}^{2}$ for several values of $N_{c}$, where it becomes clear that they are certainly well-localized functions. Here it is important to recall that the main difference between our WL function and a "true" Wannier function results from the fact that only the former depends on the filling factor, i.e., the number of particles at each site, as seen in Ref. [14]. One may also write the above stationary states in terms of these localized functions,

$$
\psi_{n}(\mathbf{r})=\frac{1}{\sqrt{N_{c}}} \sum_{k} w_{k}(r, \theta) e^{i n \theta_{k}},
$$

which will be useful to describe the dynamics we are interested in.

\section{B. Hopping and on-site energy parameters}

The $N_{c}$-mode dynamics will be described in terms of the following parameters:

$$
\begin{gathered}
\varepsilon=\int d^{2} \mathbf{r} w_{0}(r, \theta)\left[-\frac{\hbar^{2}}{2 m} \nabla^{2}+V_{\text {trap }}(\mathbf{r})\right] w_{0}(r, \theta), \\
J=-\int d^{2} \mathbf{r} w_{0}(r, \theta)\left[-\frac{\hbar^{2}}{2 m} \nabla^{2}+V_{\text {trap }}(\mathbf{r})\right] w_{1}(r, \theta), \\
J^{\prime}=-2 g \int d^{2} \mathbf{r} w_{0}^{3}(r, \theta) w_{1}(r, \theta), \\
U=g \int d^{2} \mathbf{r} w_{0}^{4}(r, \theta),
\end{gathered}
$$

which, due to the symmetry of the lattice, can be written without loss of generality only in terms of the $k=0$ and $k=1$ sites. We want to mention that these parameters can also be efficiently evaluated through the alternative formulas given in Ref. [14].

\section{TWO-MODE DYNAMICAL EQUATIONS}

When the trapping potential consists of a double well, the condensate dynamics may be simply described through a pair of coupled equations, which correspond to the two-mode model. Such a TM dynamics has been extensively studied in recent years [2]. Particularly, an improved version of this model [3] has been also applied to particles exhibiting a dipolar interaction, which generates self-induced Josephson junctions in a similar toroidal geometry [7].

\section{A. Dynamical equations in terms of the coefficients of well-localized functions}

The commonly used ansatz for the TM wave function reads

$$
\psi_{\mathrm{TM}}(r, \theta, t)=b_{R}(t) \psi_{R}(r, \theta)+b_{L}(t) \psi_{L}(r, \theta),
$$

where $\psi_{R}(r, \theta)$ and $\psi_{L}(r, \theta)$ are well-localized functions at the right and left wells, respectively. Such wave functions are easily identified with the WL functions, namely, $\psi_{R}(r, \theta)=$ $w_{0}(r, \theta)$ and $\psi_{L}(r, \theta)=w_{1}(r, \theta)$, since from Eq. (4) we get, for $N_{c}=2$,

$$
\begin{aligned}
& w_{0}(r, \theta)=\frac{1}{\sqrt{2}}\left[\psi_{0}(r, \theta)+\psi_{1}(r, \theta)\right], \\
& w_{1}(r, \theta)=\frac{1}{\sqrt{2}}\left[\psi_{0}(r, \theta)-\psi_{1}(r, \theta)\right],
\end{aligned}
$$

which turns out to be identical to the standard TM variational proposal [1].

Note that the first excited state is an odd function of $x$, as required by the TM model (antisymmetric solution). This can be easily verified by noting that the stationary state with 

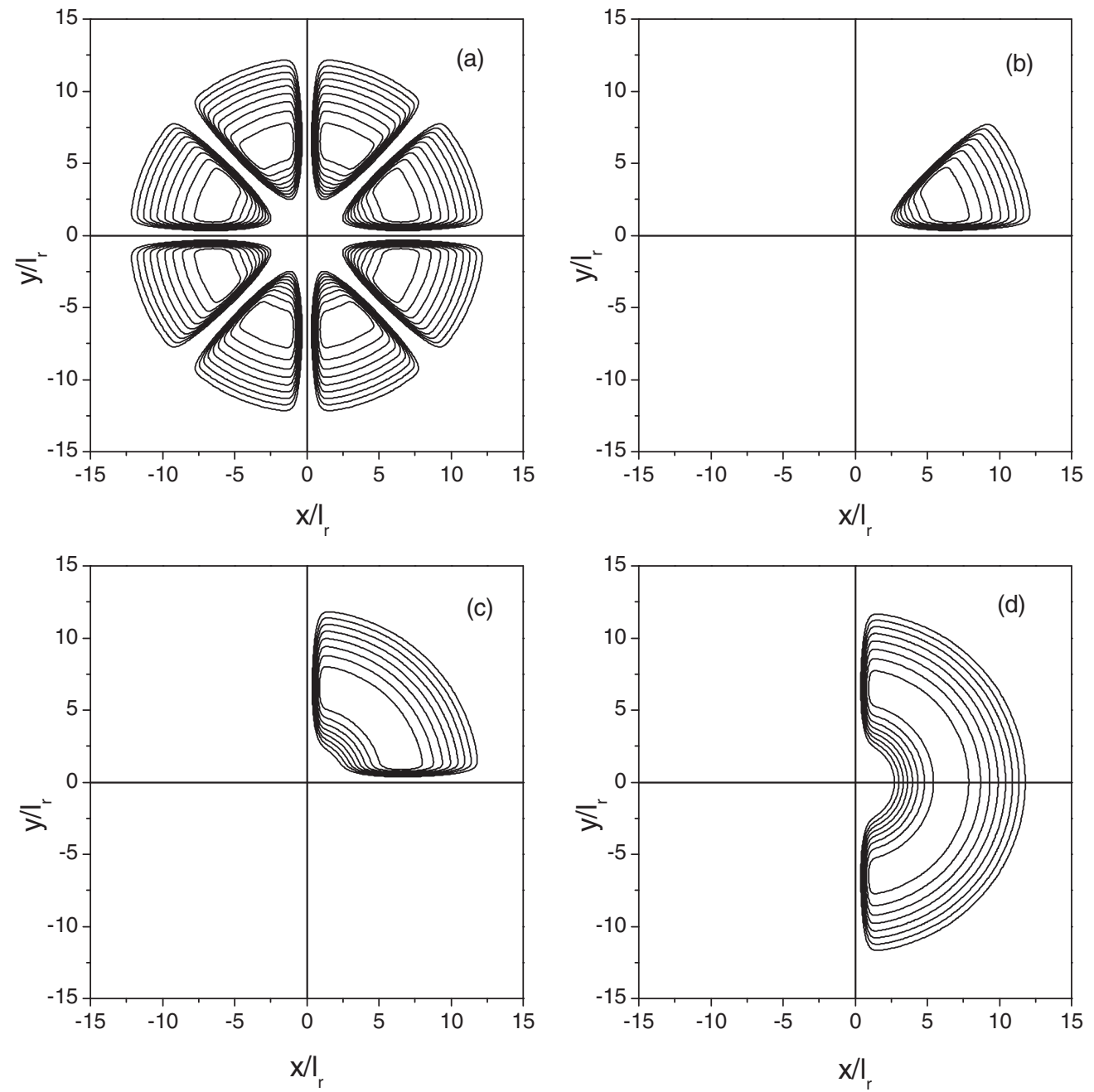

FIG. 1. Density isocontours of (a) the ground-state wave function $\left|\psi_{0}\right|^{2}$ for $N_{c}=8$ and of the WL function $w_{0}^{2}(\mathbf{r})$ for the following numbers of sites: (b) $N_{c}=8$, (c) $N_{c}=4$, and (d) $N_{c}=2$.

winding number $n=1$ has uniform phases at the right and left wells with values $\phi=0$ and $\phi=\pi$, respectively [20]. Here we may recall that this only occurs in the regime of large barriers [14], where $\psi_{1}(\mathbf{r})=\psi_{-1}(\mathbf{r})$ may be taken as a real function that does not carry any angular momentum and hence does not correspond to a "vortex" state [20].

In order to obtain the TM equations, we may replace the order parameter, which is written in terms of the WL functions according to the ansatz (10),

$$
\psi_{\mathrm{TM}}(r, \theta, t)=b_{0}(t) w_{0}(r, \theta)+b_{1}(t) w_{1}(r, \theta)
$$

in the time-dependent GP equation,

$$
\begin{aligned}
i \hbar \frac{\partial \psi_{\mathrm{TM}}(r, \theta, t)}{\partial t}= & {\left[-\frac{\hbar^{2}}{2 m} \nabla^{2}+V_{\mathrm{trap}}(r, \theta)\right.} \\
& \left.+g N\left|\psi_{\mathrm{TM}}(r, \theta, t)\right|^{2}\right] \psi_{\mathrm{TM}}(r, \theta, t) .
\end{aligned}
$$

Making use of the orthonormality of the WL functions and recalling the definitions of hopping and on-site energy parameters [Eqs. (6) to (9)], one obtains

$i \hbar \frac{d b_{0}}{d t}=\varepsilon b_{0}-J b_{1}+U N\left|b_{0}\right|^{2} b_{0}-\frac{J^{\prime}}{2} N\left[2 \operatorname{Re}\left(b_{0}^{*} b_{1}\right) b_{0}+b_{1}\right]$,

$i \hbar \frac{d b_{1}}{d t}=\varepsilon b_{1}-J b_{0}+U N\left|b_{1}\right|^{2} b_{1}-\frac{J^{\prime}}{2} N\left[2 \operatorname{Re}\left(b_{1}^{*} b_{0}\right) b_{1}+b_{0}\right]$.

The above equations correspond to the improved TM model developed in Ref. [3] and applied in Refs. [6,7]. Here it is worth noticing that we have disregarded in our derivation terms of the order of the integral

$$
I=g N \int d^{2} \mathbf{r} w_{0}^{2}(r, \theta) w_{1}^{2}(r, \theta)
$$

since the corresponding contributions have been shown to be negligible, as has also been argued in Ref. [3] for high barriers. 


\section{B. Dynamical equations in terms of the particle imbalance and phase difference}

A more convenient set of variables is obtained by observing that $b_{k}(t)=\left|b_{k}(t)\right| e^{i \phi_{k}(t)}$, where $\phi_{k}(t)$ is the uniform phase of the $k$ site and $n_{k}=N_{k}(t) / N=\left|b_{k}(t)\right|^{2}$ denotes the corresponding filling factor. Following the procedure of Ref. [7], the equations of motion for the conjugate coordinates, namely, imbalance $Z=n_{0}-n_{1}$ and phase difference $\varphi=\phi_{1}-\phi_{0}$, read

$$
\begin{gathered}
\frac{d Z}{d t}=-\sqrt{1-Z^{2}} \sin \varphi \\
\frac{d \varphi}{d t}=\Lambda_{\mathrm{eff}} Z+\left[\frac{Z}{\sqrt{1-Z^{2}}}\right] \cos \varphi
\end{gathered}
$$

where the time $t$ (in the derivatives) has been expressed in units of $\hbar / 2 J_{\text {eff }}$ and we have defined $\Lambda_{\text {eff }}=\frac{U N}{2 J_{\text {eff }}}$, with $J_{\text {eff }}=J+$ $\frac{J^{\prime}}{2} N$. Note that the above equations possess the same structure as the standard TM ones, except that the bare $J$ has been replaced by an effective hopping parameter $J_{\text {eff }}$, which takes into account the interaction between particles. It is interesting to recall that $J$ may be negative, as occurs in the present calculations, while $J_{\text {eff }}$ remains always positive [14].

The TM equations (18) and (19) can also be derived from the "classical" Hamiltonian

$$
H(Z, \varphi)=\frac{1}{2} \Lambda_{\text {eff }} Z^{2}-\sqrt{1-Z^{2}} \cos \varphi
$$

since we have

$$
\frac{d Z}{d t}=-\frac{\partial H}{\partial \varphi}, \quad \frac{d \varphi}{d t}=\frac{\partial H}{\partial Z} .
$$

For low $\Lambda_{\text {eff }}$ values the Hamiltonian exhibits only a minimum at $(Z, \varphi)=(0,0)$, and the dynamics becomes restricted to Josephson-type oscillations. For $\Lambda_{\text {eff }}>1$ a maximum appears at $\varphi=\pi$ and

$$
Z_{M}=\sqrt{1-\frac{1}{\Lambda_{\mathrm{eff}}^{2}}}
$$

which gives rise to a self-trapping regime. Around this maximum the orbits are restricted to only one sign of the imbalance. In other words, if one starts with a positive imbalance, it always remains positive. A ST running-phase mode [1] arises for $\Lambda_{\text {eff }}>2$, which is characterized by an unbounded $\varphi$ value. To find the value $Z_{c}$ above which the dynamics becomes ST for $\varphi(t=0)=0$, we need to impose the condition $H\left(Z_{c}, 0\right)=H(0, \pi)$, which yields

$$
Z_{c}=2 \frac{\sqrt{\Lambda_{\mathrm{eff}}-1}}{\Lambda_{\mathrm{eff}}} .
$$

In this work we are interested in the range $\Lambda_{\text {eff }} \gg 1$, and thus a small $Z_{c}$ value is attained. In fact, we have calculated the values of on-site energy and hopping parameters, $U=6.73 \times 10^{-4} \hbar \omega_{r}, J=-3.66 \times 10^{-4} \hbar \omega_{r}$, and $\frac{J^{\prime}}{2} N=5.05 \times 10^{-4} \hbar \omega_{r}$, from which we obtained the TM parameters, $J_{\text {eff }}=1.39 \times 10^{-4} \hbar \omega_{r}$ and $\Lambda_{\text {eff }}=2.42 \times 10^{5}$.

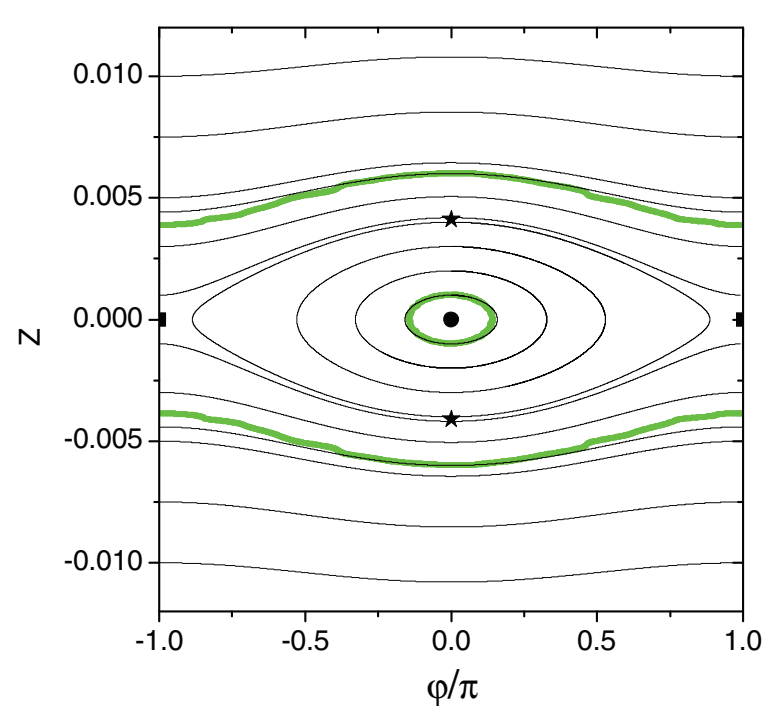

FIG. 2. (Color online) Phase diagram of the improved TM model for imbalance $Z$ and phase difference $\varphi$. The circle and squares respectively indicate the positions of the minimum and saddle points of the Hamiltonian (20), while the stars correspond to the critical value $\left|Z_{c}\right|$ [Eq. (23)]. We have also depicted as thick green solid lines the results of the GP simulation for the initial conditions $(|Z|, \varphi)=$ $(0.001,0)$ and $(|Z|, \varphi)=(0.006,0)$.

In addition, we have found $I=6.05 \times 10^{-7} \hbar \omega_{r}$ [Eq. (17)], which justifies having neglected terms proportional to such a parameter in the equations.

In Fig. 2 we show the phase diagram $(Z, \varphi)$ for $|Z|<0.012$ since for larger values of $|Z|$ the orbits are almost horizontal. The thick green lines correspond to exact numerical evolutions for the initial conditions: $(|Z|, \varphi)=(0.001,0)$ (Josephson) and $(|Z|, \varphi)=(0.006,0)(\mathrm{ST})$. To obtain such simulations, we have solved the time-dependent GP equation with an initial wave function which reproduces the same initial condition assumed for the TM model evolution. In order to compare the phase differences of both results, we have averaged the GP phase in each $k$ well according to

$$
\phi_{k}=\int d^{2} \mathbf{r} w_{k}^{2}(\mathbf{r}) \phi(\mathbf{r}),
$$

where $\phi(\mathbf{r})$ denotes the phase of the GP wave function. We want to note that the Bloch states of Ref. [20] correspond to equally populated wells with different winding numbers. In the double-well potential these states are represented by the stationary points located at $Z=0$ in Fig. 2. The minimum at $\varphi=0$ corresponds to a vanishing winding number, while the saddle point at $|\varphi|=\pi$ corresponds to winding numbers with $|n|=1$. The latter, however, does not correspond to a vortex state since it possesses zero angular momentum, as discussed in Ref. [20].

Typical time evolutions of Josephson oscillations and ST orbits are shown in Figs. 3 and 4, respectively. In Fig. 3 we depict $Z$ and $\varphi$ as functions of time for the GP simulations (solid line), together with the results arising from the TM model (dot-dashed blue line). On the other hand, Fig. 4 shows the same evolutions for a larger initial imbalance, where we clearly observe a self-trapping behavior. In both 

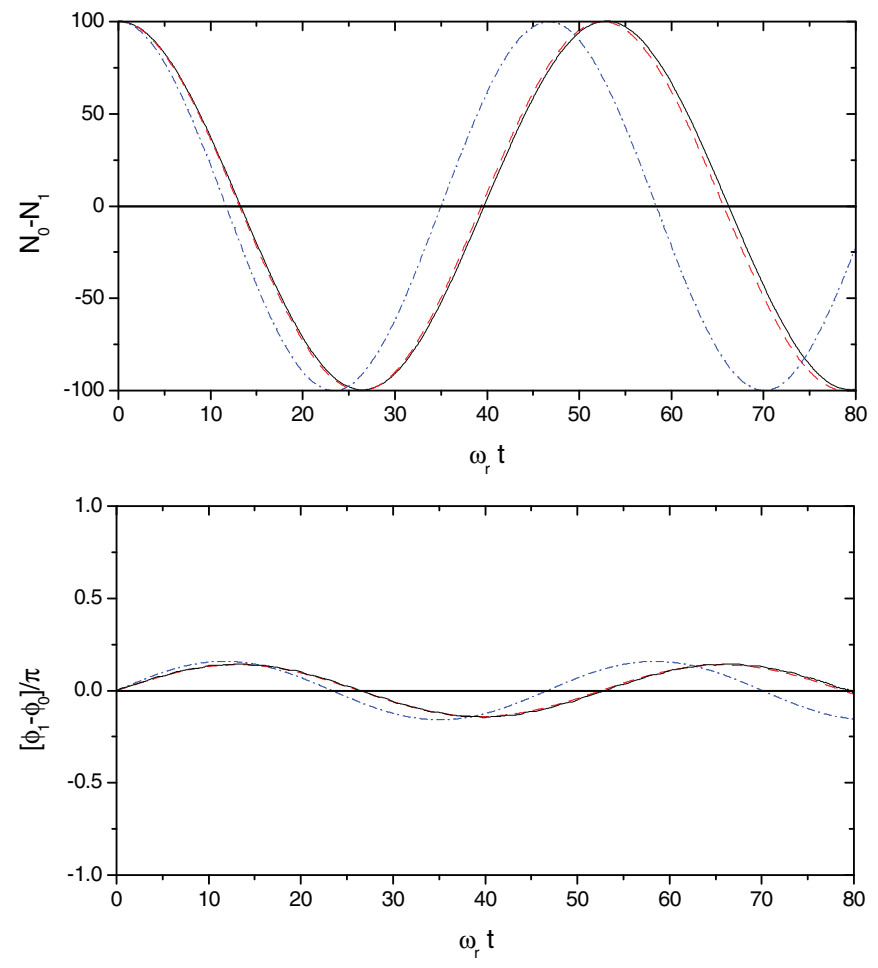

FIG. 3. (Color online) Josephson oscillation in the double-well system with an initial imbalance $Z=10^{-3}$. (top) Imbalance and (bottom) phase difference are depicted as functions of time. The solid line corresponds to the GP simulation, while the dot-dashed blue and dashed red lines correspond to TM evolutions with $U=6.73 \times 10^{-4} \hbar \omega_{r}$ and a renormalized on-site energy parameter $U_{R}=5.28 \times 10^{-4} \hbar \omega_{r}$, respectively.
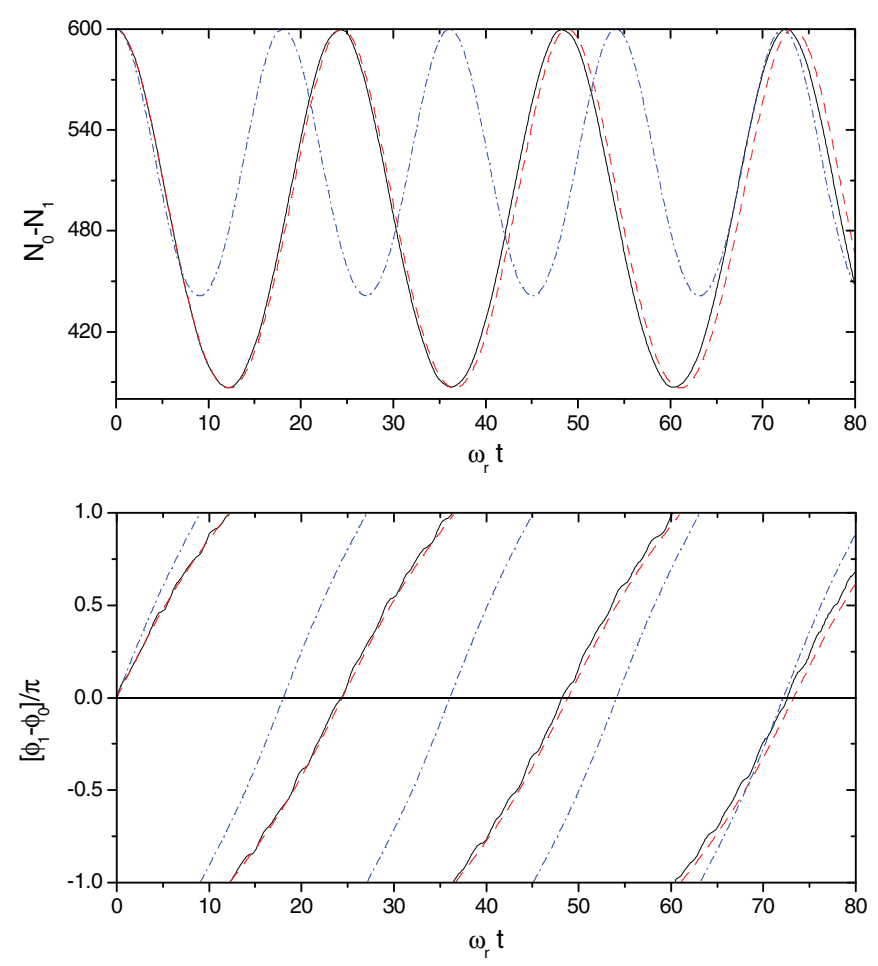

FIG. 4. (Color online) Same as Fig. 3 for a self-trapping evolution with an initial imbalance $Z=6 \times 10^{-3}$. cases we may see that the TM model predicts a faster dynamics than the GP simulation. As we will show in the next section, such a discrepancy can be substantially reduced when using a renormalized on-site interaction energy parameter (dashed red line).

\section{Characteristic times}

In this section we will derive a formula for the period of ST oscillations. Before this, we recall that the Josephson period in the limit of small oscillations reads $[1,6]$

$$
T_{\text {so }}=\frac{\pi \hbar}{J_{\text {eff }} \sqrt{\Lambda_{\text {eff }}+1}} .
$$

Replacing in the above equation the values of Sec. IV B, we obtain $T_{\text {so }}=46.0 \omega_{r}^{-1}$. This is a rather good estimate of the TM period in Fig. $3\left(T_{\mathrm{TM}}=46.68 \omega_{r}^{-1}\right)$, but it clearly underestimates the corresponding GP period $\left(T_{\mathrm{GP}} \simeq 53.09 \omega_{r}^{-1}\right)$.

On the other hand, the phase difference increases almost linearly with time for small-imbalance oscillations in the ST regime,

$$
\varphi(t) \simeq \frac{2 \pi}{T_{\mathrm{ST}}} t,
$$

as seen in Fig. 4. Now, to be consistent with this approximation, we first rewrite Eq. (19) without the adimensionalized time and next approximate such an expression for $\Lambda_{\text {eff }}=\frac{U N}{2 J_{\text {eff }}} \gg 1$ and $|Z| \ll 1$ as follows:

$$
\begin{aligned}
\frac{d \varphi}{d t} & =\frac{N U}{\hbar} Z+\frac{2 J_{\mathrm{eff}}}{\hbar}\left[\frac{Z}{\sqrt{1-Z^{2}}}\right] \cos \varphi \\
& \simeq \frac{N U}{\hbar} Z \simeq \frac{N U}{\hbar} Z_{0}
\end{aligned}
$$

where $Z_{0}=\overline{Z(t)}$ denotes the mean value of the timedependent imbalance. Note in Fig. 4 that the maximum departure of $Z(t)$ from such a mean value lies within $20 \%$. Therefore, from (26) and (27) we may estimate the ST period as

$$
T_{\mathrm{ST}}=\frac{2 \pi \hbar}{U \overline{\Delta N}},
$$

where $\overline{\Delta N}=Z_{0} N$ denotes the time average of the particle number difference between sites. If we calculate such an average from the TM model of Fig. 4, we obtain $\overline{\Delta N} \simeq 520$, from which Eq. (28) yields $T_{\mathrm{ST}}=17.94 \omega_{r}^{-1}$, which is a good estimate of the TM period of $18.04 \omega_{r}^{-1}$ in Fig. 4. Now, given that the TM dynamics turns out to be noticeably faster than the GP evolution, while conserving the shape, it suggests that a renormalized value of $U$ in (28) could heal this mismatch. In fact, with $T_{\mathrm{GP}}=24.1 \omega_{r}^{-1}$ and $\overline{\Delta N}_{\mathrm{GP}}=494$, we may propose to replace $U$ in Eq. (28) by the following renormalized on-site interaction energy parameter:

$$
U_{R}=\frac{2 \pi \hbar}{T_{\mathrm{GP}} \overline{\Delta N}_{\mathrm{GP}}}=5.28 \times 10^{-4} \hbar \omega_{r} .
$$

Thus, we have repeated the numerical calculations of the TM model with the above parameter, finding excellent agreement 

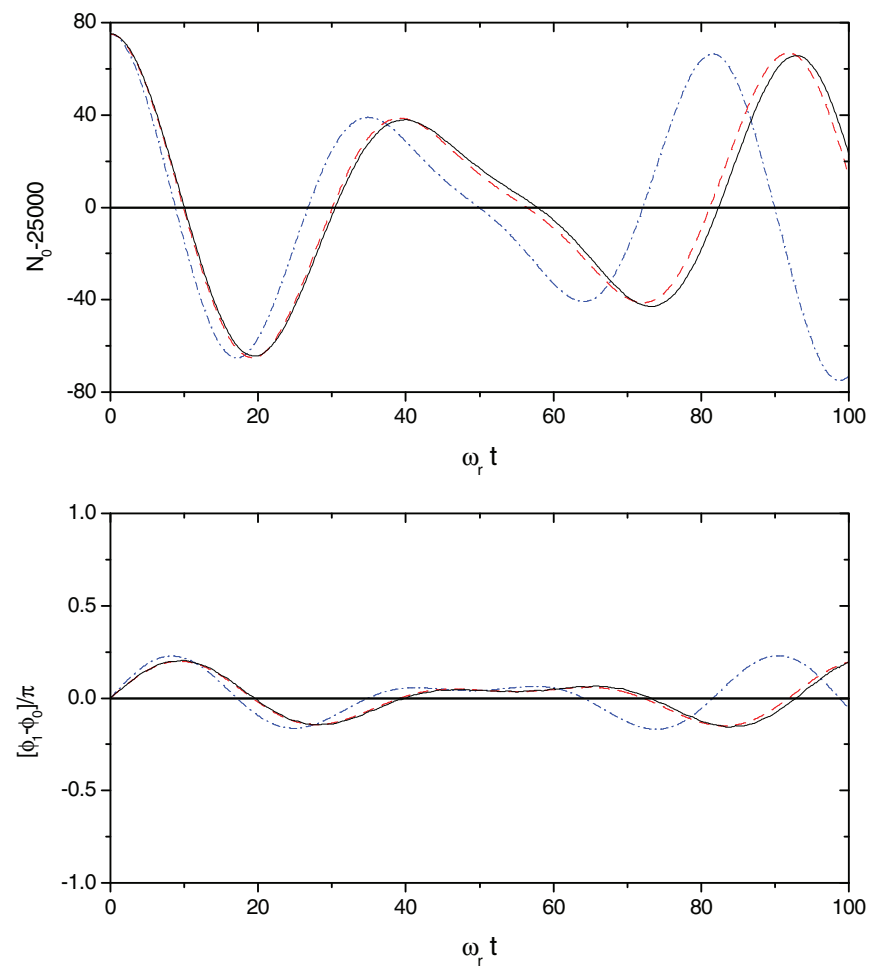

FIG. 5. (Color online) (top) Time evolution of population $N_{0}-M$ and (bottom) phase difference $\phi_{1}-\phi_{0}$ for the four-well system and initial conditions $N_{0}-M=75, N_{k}-M=-25(k=$ $1,2,3)$ with a uniformly vanishing phase. The solid line corresponds to the GP simulation, while the dot-dashed blue and dashed red lines correspond to the MM and RMM models, with on-site interaction energy parameters $U=1.38 \times 10^{-3} \hbar \omega_{r}$ and $U_{R}=1.08 \times 10^{-3} \hbar \omega_{r}$, respectively.

with the GP results, as clearly observed in Figs. 3 and 4. It is also remarkable that the period for small Josephson oscillations (25) gets now closer to the GP value when using the renormalized parameter (29) $\left(T_{\text {so }}=51.5 \omega_{r}^{-1}\right)$. Therefore, a more accurate Hamiltonian (20) can be constructed by replacing $U$ with $U_{R}$ in $\Lambda_{\text {eff }}$.

\section{MULTIPLE-MODE DYNAMICAL EQUATIONS}

The two-mode equations describing the boson Josephsonjunction dynamics of two weakly coupled Bose-Einstein condensates [2], along with their recent improvements for high particle numbers $[3,4]$, can be generalized to multiplemode dynamical equations for $N_{c}$ Bose-Einstein condensates forming a ring. In fact, we look for a solution of the timedependent GP equation

$$
\begin{aligned}
i \hbar \frac{\partial \psi_{\mathrm{MM}}(r, \theta, t)}{\partial t}= & {\left[-\frac{\hbar^{2}}{2 m} \nabla^{2}+V_{\text {trap }}(r, \theta)\right.} \\
& \left.+g N\left|\psi_{\mathrm{MM}}(r, \theta, t)\right|^{2}\right] \psi_{\mathrm{MM}}(r, \theta, t)
\end{aligned}
$$

within the variational ansatz

$$
\psi_{\mathrm{MM}}(r, \theta, t)=\sum_{k=0}^{N_{c}-1} b_{k}(t) w_{k}(r, \theta),
$$
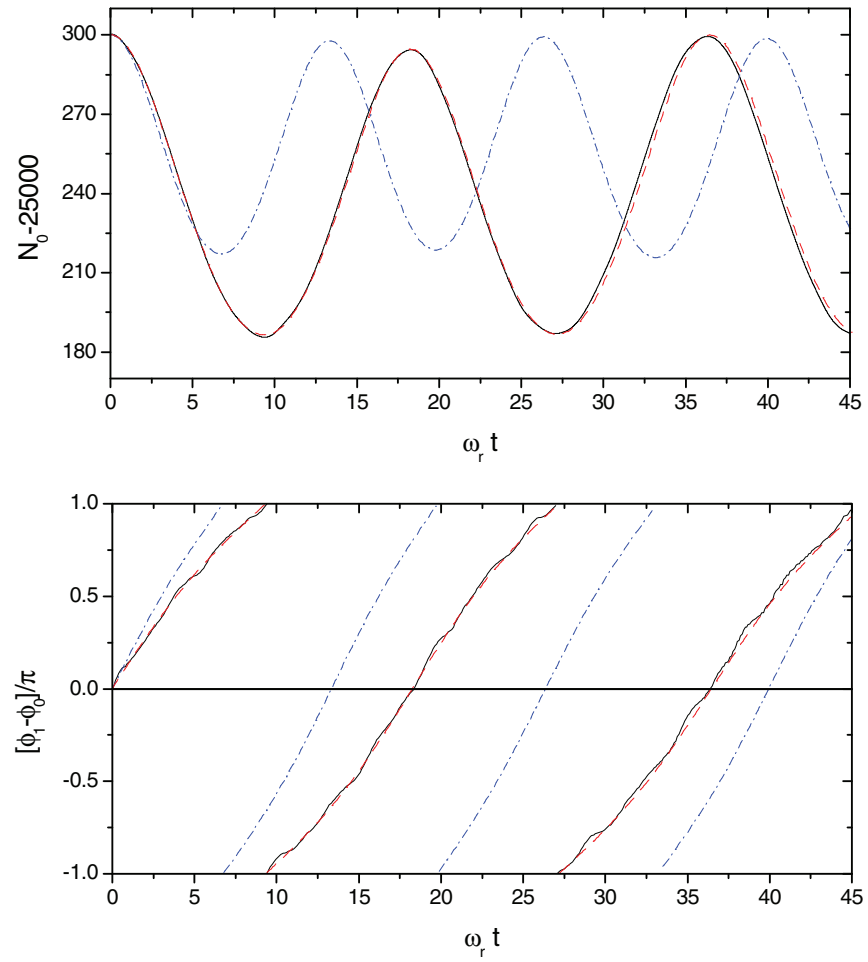

FIG. 6. (Color online) Same as Fig. 5 for initial conditions $N_{0}-M=300$ and $N_{k}-M=-100(k=1,2,3)$.

where the phase of the time-dependent complex amplitude $b_{k}$ corresponds to the uniform phase of the order parameter at the $k$ th site, while $N\left|b_{k}\right|^{2}$ yields the site population. Then, replacing (31) in (30) and making use of the orthonormality of the set of WL functions, we may extract the following system of $N_{c}$ nonlinear equations:

$$
\begin{aligned}
i \hbar \frac{d b_{k}}{d t}= & \varepsilon b_{k}-J\left(b_{k-1}+b_{k+1}\right)+U N\left|b_{k}\right|^{2} b_{k} \\
& -\frac{J^{\prime}}{2} N\left\{2 \operatorname{Re}\left[b_{k}^{*}\left(b_{k-1}+b_{k+1}\right)\right] b_{k}\right. \\
& \left.+\left(\left|b_{k}\right|^{2}+\left|b_{k-1}\right|^{2}\right) b_{k-1}+\left(\left|b_{k}\right|^{2}+\left|b_{k+1}\right|^{2}\right) b_{k+1}\right\} .
\end{aligned}
$$

Note that the above expression assumes that each site $k$ is surrounded by two different neighbors $k-1$ and $k+1$; for that reason the $N_{c}=2$ case has been treated separately. In addition, the site denoted by $k=N_{c}(k=-1)$ must be identified with that of $k=0\left(k=N_{c}-1\right)$. If we use $b_{k}=\left|b_{k}\right| e^{i \phi_{k}}$, the time derivative in (32) reads

$$
i \hbar \frac{d b_{k}}{d t}=\hbar\left(i \frac{d\left|b_{k}\right|}{d t}-\left|b_{k}\right| \frac{d \phi_{k}}{d t}\right) e^{i \phi_{k}}
$$

Next, replacing (33) in (32), multiplying this equation by $e^{-i \phi_{k}}$, and separating the real and imaginary parts, one can, after some algebra, decouple Eq. (32) into the following $2 N_{c}$ real equations, written in terms of population $n_{k}=\left|b_{k}\right|^{2}=N_{k} / N$ 
and phase difference $\varphi_{k}=\phi_{k}-\phi_{k-1}$ :

$$
\begin{aligned}
\hbar \frac{d n_{k}}{d t}= & -2 J\left[\sqrt{n_{k} n_{k+1}} \sin \varphi_{k+1}-\sqrt{n_{k} n_{k-1}} \sin \varphi_{k}\right]-J^{\prime} N\left[\sqrt{n_{k} n_{k+1}}\left(n_{k}+n_{k+1}\right) \sin \varphi_{k+1}-\sqrt{n_{k} n_{k-1}}\left(n_{k}+n_{k-1}\right) \sin \varphi_{k}\right] \\
\hbar \frac{d \varphi_{k}}{d t}= & U N\left(n_{k-1}-n_{k}\right)-J\left[\left(\sqrt{\frac{n_{k}}{n_{k-1}}}-\sqrt{\frac{n_{k-1}}{n_{k}}}\right) \cos \varphi_{k}+\sqrt{\frac{n_{k-2}}{n_{k-1}}} \cos \varphi_{k-1}-\sqrt{\frac{n_{k+1}}{n_{k}}} \cos \varphi_{k+1}\right] \\
& -\frac{J^{\prime} N}{2}\left[\left(n_{k} \sqrt{\frac{n_{k}}{n_{k-1}}}-n_{k-1} \sqrt{\frac{n_{k-1}}{n_{k}}}\right) \cos \varphi_{k}+\left(3 \sqrt{n_{k-2} n_{k-1}}+n_{k-2} \sqrt{\frac{n_{k-2}}{n_{k-1}}}\right) \cos \varphi_{k-1}\right. \\
& \left.-\left(3 \sqrt{n_{k+1} n_{k}}+n_{k+1} \sqrt{\frac{n_{k+1}}{n_{k}}}\right) \cos \varphi_{k+1}\right] .
\end{aligned}
$$

The above MM dynamical equations constitute the generalization of the TM pair of equations (18) and (19) for $N_{c}>2$. Note that similar to the TM case, only $2 N_{c}-2$ of the above equations are independent since the variables must fulfill $\sum_{k} n_{k}=1$ and $\sum_{k} \varphi_{k}=0$.
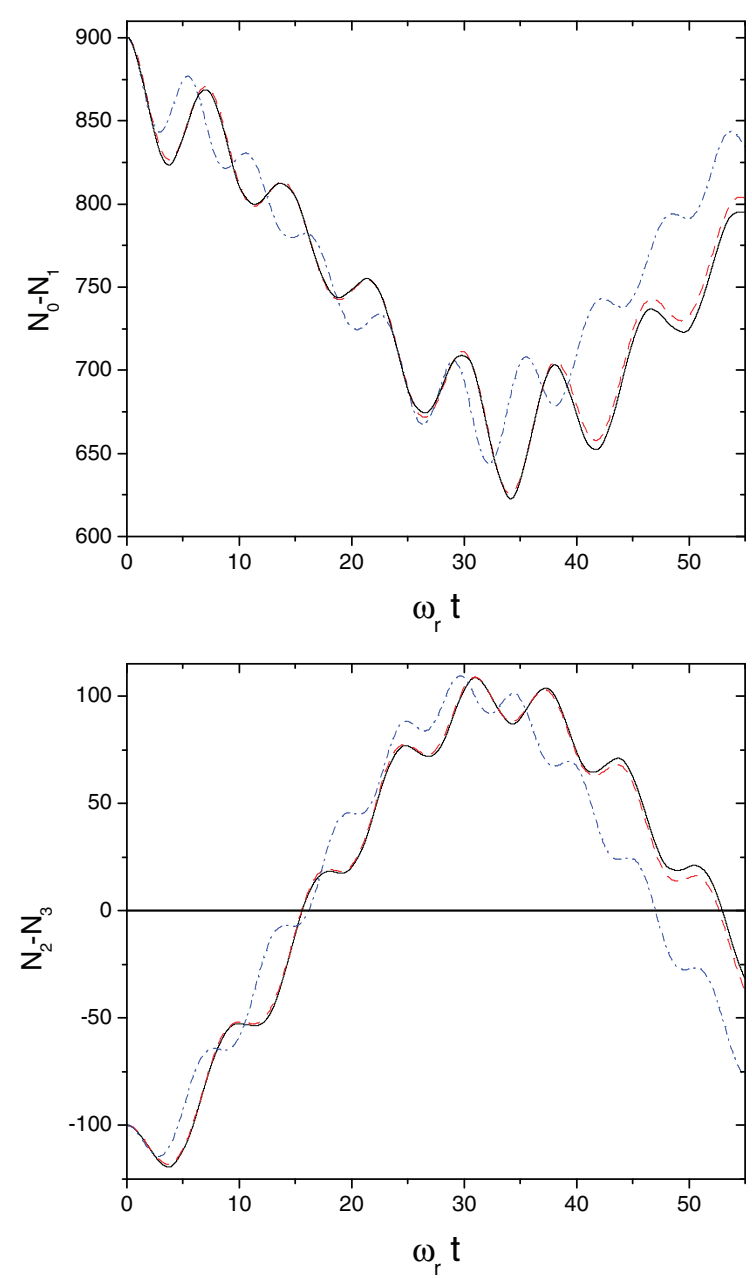

\section{A. Four-well ring lattice}

In order to compare the MM dynamics with the results of GP simulations, we have numerically integrated the system (34) and (35) for $N_{c}=4$ and two initial configurations. The model parameters utilized in this case were
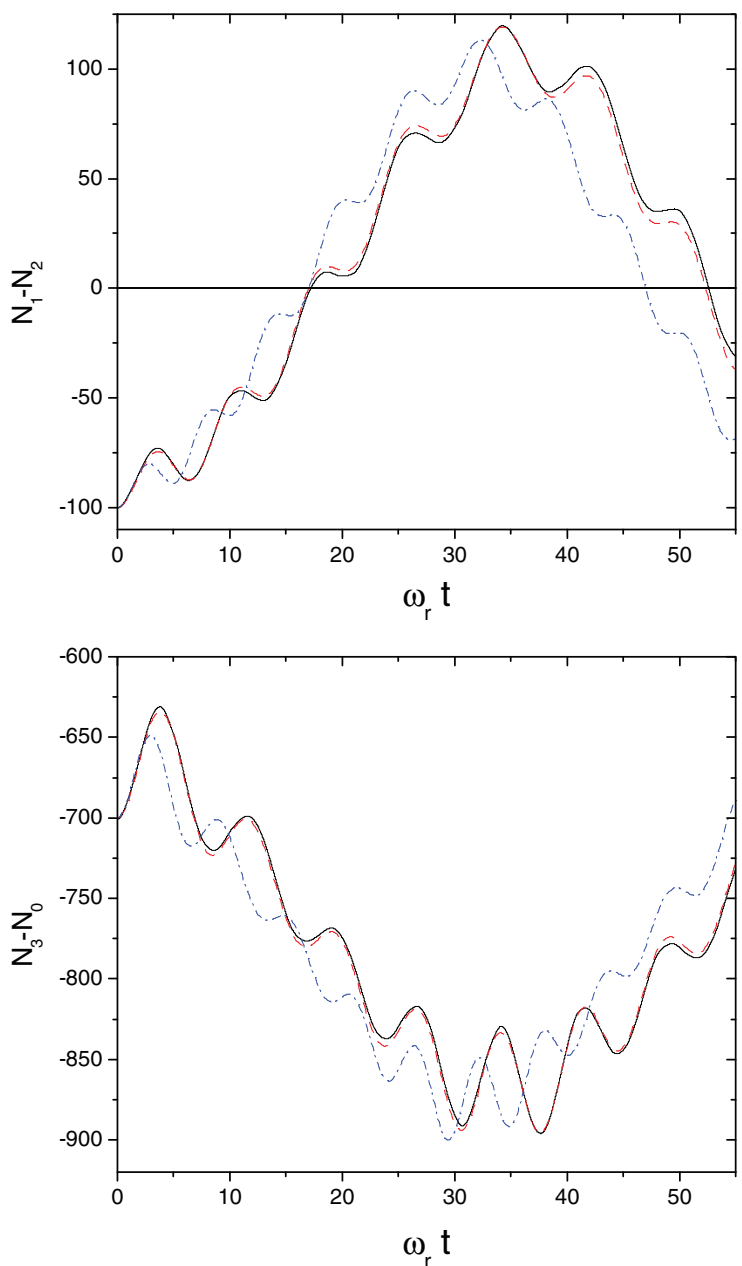

FIG. 7. (Color online) Particle number differences between neighboring sites for $N_{c}=4$ and the initial conditions $N_{0}-M=600$, $N_{1}-M=-300, N_{2}-M=-200$, and $N_{3}-M=-100$. The solid line corresponds to the GP simulation, while the dot-dashed blue and dashed red lines correspond to the MM and RMM models, with on-site interaction energy parameters $U=1.38 \times 10^{-3} \hbar \omega_{r}$ and $U_{R}=1.08 \times 10^{-3} \hbar \omega_{r}$, respectively. 
$U=1.38 \times 10^{-3} \hbar \omega_{r}, \quad J=-4.98 \times 10^{-4} \hbar \omega_{r}, \quad$ and $\quad J^{\prime}=$ $2.76 \times 10^{-8} \hbar \omega_{r}$.

\section{Symmetric case}

Here we consider initial conditions which are symmetric with respect to the right and left from the $k=0$ well, as also studied by De Liberato and Foot [13]. Particularly, in Fig. 5 we have chosen the following initial conditions: $N_{0}-M=75$ and $N_{k}-M=-25$ for the remaining sites, where $M=N / N_{c}=25000$ denotes the mean number of particles per site in the ground state. We may observe in the top panel that the population oscillates around the mean value $M$ without any periodicity, at least for the times involved in our numerical simulations. A similar behavior for the phase difference has been depicted in the bottom panel of Fig. 5. Note that the MM model again reproduces the shape of the GP evolution in a faster dynamics, as already observed for the TM model. Figure 6 shows the time evolution for the same symmetric initial configuration, but with a higher population in the $k=0$ well $\left(N_{0}-M=300, N_{k}-M=-100\right.$ for $k=$ $1,2,3)$. We observe in this case a clear ST regime, with the population $N_{0}-M$, which remains positive during the oscillations performed around $N_{0}-25000 \simeq 240$, and an unbounded phase that increases almost linearly with time.
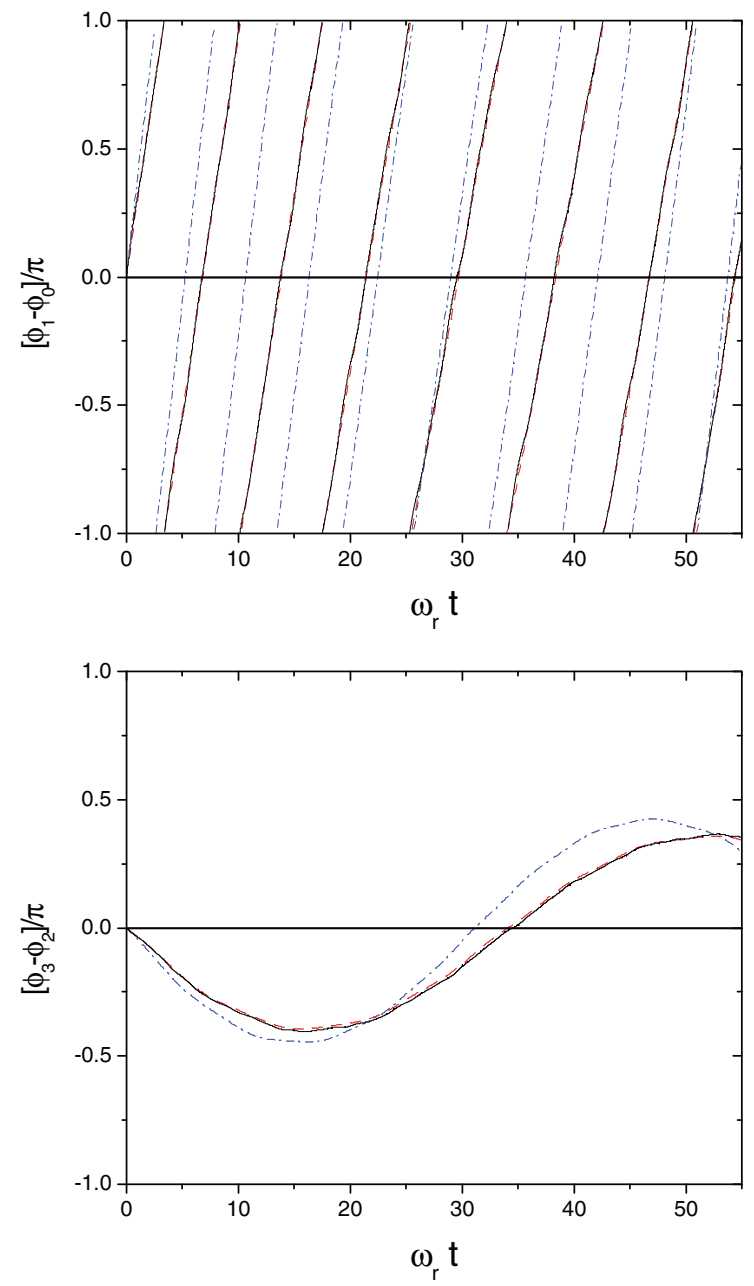

Now we will generalize the treatment of Sec. IV C to estimate the ST period in order to derive a renormalized on-site energy parameter. First, according to the bottom panel of Fig. 6 we may approximate [cf. Eq. (26)]

$$
\varphi_{1}(t) \simeq \frac{2 \pi}{T_{\mathrm{ST}}} t
$$

and next, consistent with this approximation [cf. Eq. (27)], we approximate Eq. (35) as

$$
\frac{d \varphi_{1}}{d t} \simeq \frac{U N\left(n_{0}-n_{1}\right)}{\hbar} \simeq \frac{U\left(\overline{N_{0}-N_{1}}\right)}{\hbar},
$$

where the upper bar again denotes time average. Therefore, from (36) and (37) we may estimate the ST period as

$$
T_{\mathrm{ST}} \simeq \frac{2 \pi \hbar}{U\left(\overline{N_{0}-N_{1}}\right)},
$$

which, taking into account the value $\left(\overline{N_{0}-N_{1}}\right)=345$ extracted from the MM results, yields $T_{\mathrm{ST}} \simeq 13 \omega_{r}^{-1}$, in accordance with the period of the MM model in Fig. 6 (dot-dashed blue lines). Then, we may repeat the procedure of Sec. IV C and extract a renormalized on-site interaction energy parameter,

$$
U_{R}=\frac{2 \pi \hbar}{T_{\mathrm{GP}}\left(\overline{N_{0}-N_{1}}\right)_{\mathrm{GP}}},
$$
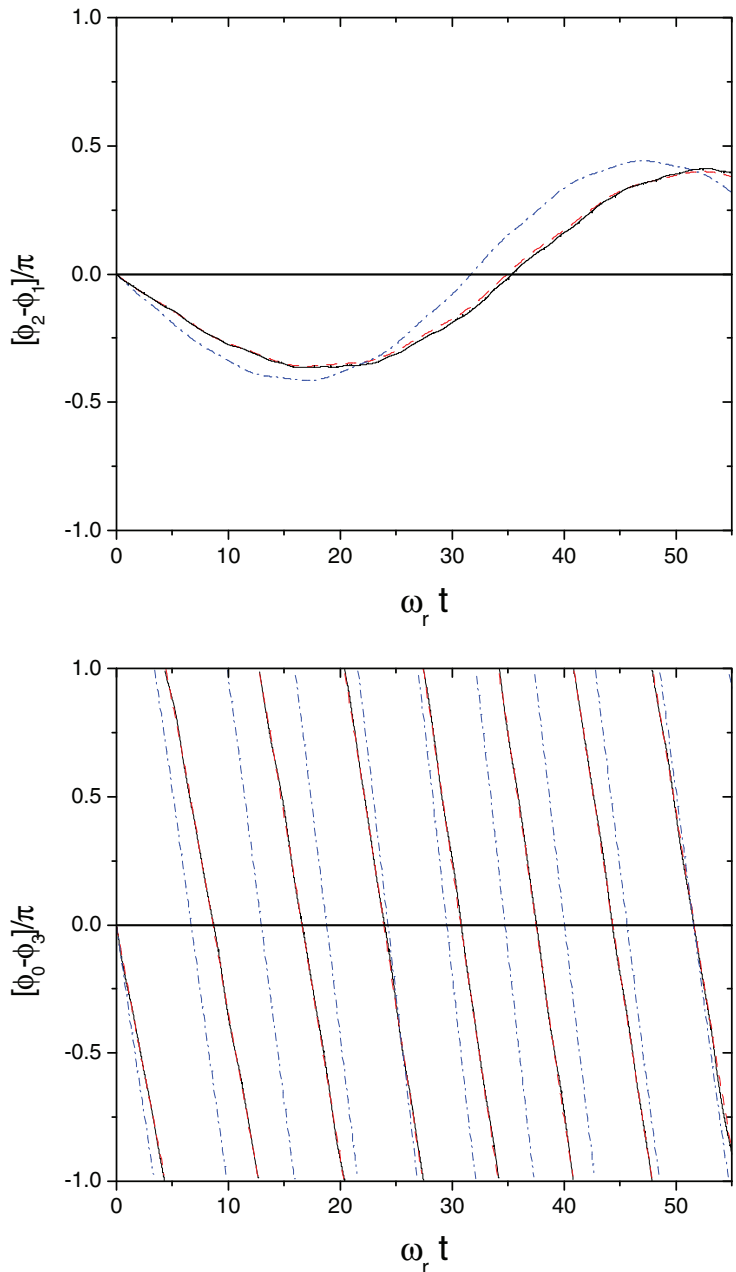

FIG. 8. (Color online) Same as Fig. 7 for the phase differences between neighboring sites. 

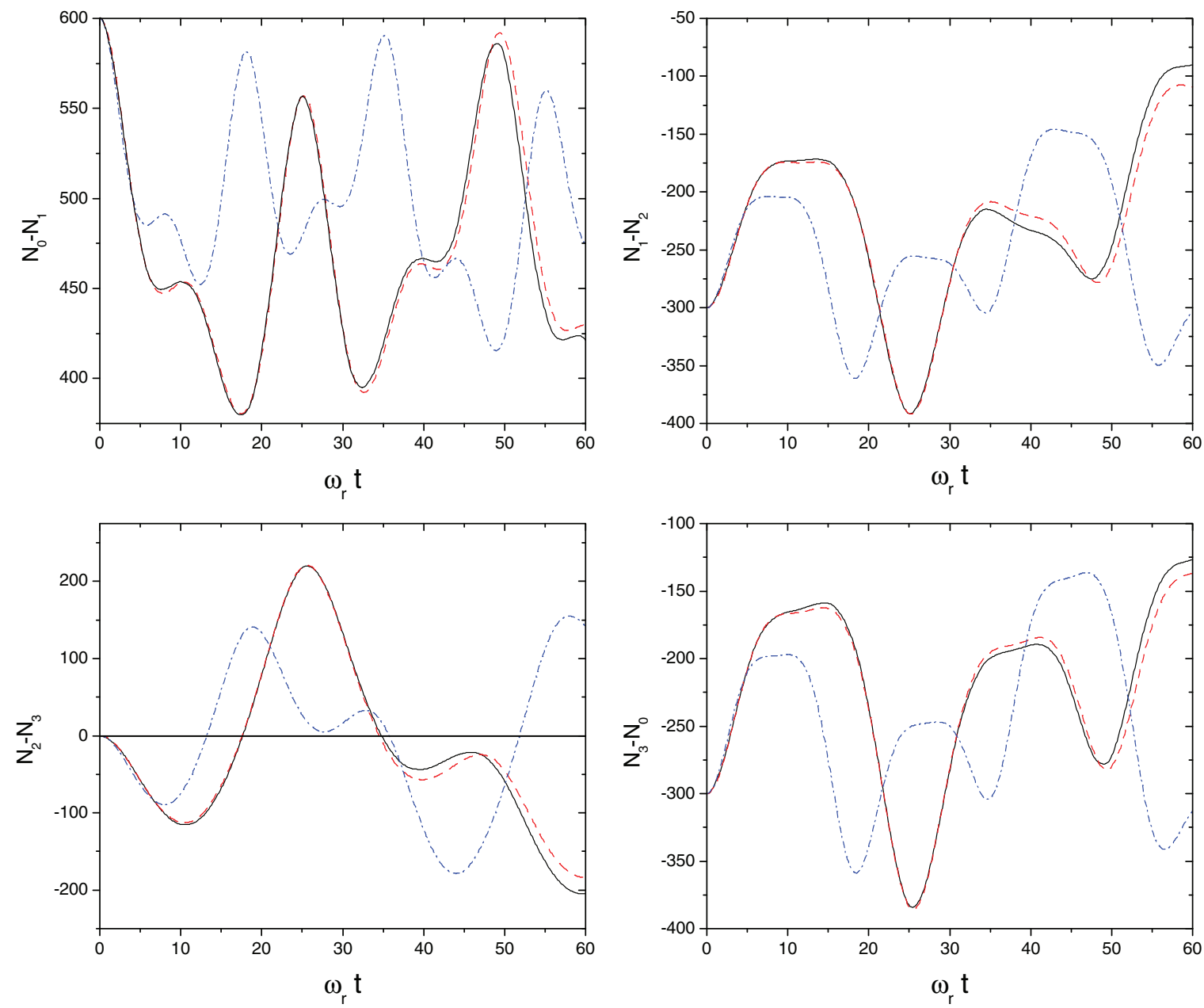

FIG. 9. (Color online) Same as Fig. 7 for initial conditions $N_{0}-M=300, N_{1}-M=-300, N_{k}-M=0(k=2,3)$.

where the values $\left(\overline{N_{0}-N_{1}}\right)_{\mathrm{GP}}=323$ and $T_{\mathrm{GP}} \simeq 18 \omega_{r}^{-1}$, arising from the GP simulation results, yield $U_{R}=1.08 \times$ $10^{-3} \hbar \omega_{r}$. The use of this renormalized $U$ parameter in the MM calculations leads to a much better agreement with the GP results, as clearly shown in Figs. 5 and 6. We will call this improved MM model the renormalized multiple-mode model.

\section{Nonsymmetric case}

To test the quality of the above RMM model, we will analyze the time evolution of two nonsymmetric initial configurations utilizing the same value for $U_{R}$ extracted in the previous section. In Figs. 7 and 8, we have plotted the population and phase differences between adjacent sites, respectively, for initial conditions $N_{0}-M=600, N_{1}-M=-300$, $N_{2}-M=-200$, and $N_{3}-M=-100$. We may observe that the RMM model fits much more accurately the GP simulation results than the original MM model. A similar improvement may be observed in Figs. 9 and 10 for the second initial conditions, $N_{0}-M=300, N_{1}-M=-300$, and $N_{k}-M=0$ for $k=2,3$. As inferred from Figs. 7 and 8 , such a configuration presents self-trapping in the $k=0$ site, while for the second initial conditions, this system exhibits self-trapping in the $k=0$ site, self-depletion in the $k=1$ site, and an irregular oscillatory dynamics around the mean number of particles on the remaining wells, as seen from
Figs. 9 and 10. The latter configuration had been previously described by means of a standard MM model by De Liberato and Foot [13].

\section{B. Eight-well ring lattice}

To conclude we will explore a ring lattice consisting of a larger number of wells, $N_{c}=8$. The corresponding MM parameters are as follows: $U=2.918 \times 10^{-3} \hbar \omega_{r}, J=$ $-1.898 \times 10^{-3} \hbar \omega_{r}$, and $J^{\prime}=2.118 \times 10^{-7} \hbar \omega_{r}$. In Fig. 11, we have depicted the population of the site $k=0$ and the phase difference between the $k=1$ and $k=0$ sites for three different initial conditions. Then, we may obtain as before a renormalized on-site energy parameter $U_{R}$ from the GP results of the ST regime depicted in the right panels of Fig. 11. In fact, replacing the $\mathrm{ST}$ period $T_{\mathrm{GP}} \simeq 8.4 \omega_{r}^{-1}$ and the average difference $\left(\overline{N_{0}-N_{1}}\right)_{\mathrm{GP}} \simeq 335$ in Eq. (39), we obtain a RMM model parameter $U_{R}=2.2 \times 10^{-3}$, which yields a sizable improvement to the MM results, as seen in Fig. 11.

\section{CALCULATION OF THE EFFECTIVE INTERACTION ENERGY PARAMETER USING THE GROUND-STATE DENSITY}

Recently, it has been demonstrated [9] that for a doublewell system, the on-site interaction energy dependence on the 

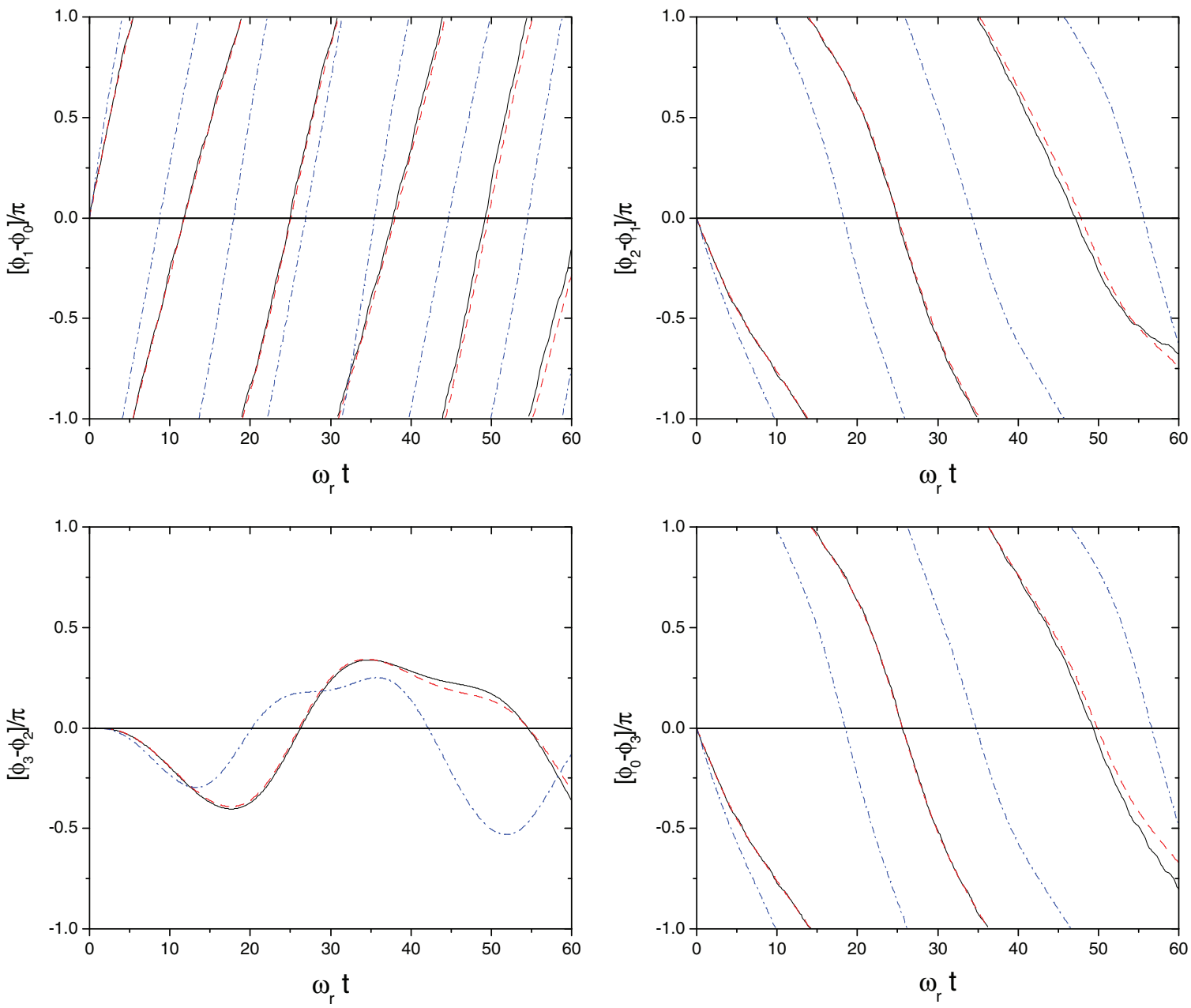

FIG. 10. (Color online) Same as Fig. 9 for the phase differences between neighboring sites.

imbalance should be taken into account in the two-mode model in order to accurately describe the exact dynamics. There, using a Thomas-Fermi density, a linear dependence with the imbalance has been analytically encountered, and this has been shown to give rise to an effective interaction energy parameter in the two-mode equations of motion. Here we generalize, beyond the Thomas-Fermi approximation, that result to the case of multiple-well configurations. Following the procedure of Ref. [9] adapted to $N_{c}$ wells and using numerically obtained densities, we have to evaluate the quotient

$$
\frac{U_{k}}{U} \simeq \frac{\int d^{2} \mathbf{r} \rho_{N}(\mathbf{r}) \rho_{N+\Delta N}(\mathbf{r})}{\int d^{2} \mathbf{r} \rho_{N}^{2}(\mathbf{r})},
$$

where we have further assumed in (40) that instead of localized on-site densities, we may use the ground-state densities $\rho_{N}(\mathbf{r})$ and $\rho_{N+\Delta N}(\mathbf{r})$ normalized to unity of systems with $N$ and $N+\Delta N$ particles, respectively, with $\Delta N=N_{c} \Delta N_{k}=$ $N_{c} N_{k}-N$.

Numerical calculations of the right-hand side of (40) are depicted in Fig. 12, where we may observe a linear behavior with $N_{c} \Delta N_{k} / N$ for different numbers of lattice sites. Note that the apparent counterintuitive decrease of this function with the site population is related to the fact that the densities must be normalized to unity.
Taking into account this linear dependence of the on-site energy parameter we may write

$$
\frac{U_{k}}{U} \simeq 1-\alpha \frac{N_{c} \Delta N_{k}}{N},
$$

where the values of $\alpha$ in Table I correspond to the linear fits of the points in Fig. 12. To include this correction in the MM model we must evaluate [9]

$$
\begin{aligned}
\frac{U_{k-1}}{U} N_{k-1}-\frac{U_{k}}{U} N_{k}= & \left(1-\alpha \frac{N_{c} \Delta N_{k-1}}{N}\right)\left(\Delta N_{k-1}+\frac{N}{N_{c}}\right) \\
& -\left(1-\alpha \frac{N_{c} \Delta N_{k}}{N}\right)\left(\Delta N_{k}+\frac{N}{N_{c}}\right),
\end{aligned}
$$

TABLE I. Linear correction coefficient $\alpha$ of the on-site interaction energy parameter of the $k$ site [Eq. (41)] and effective $(\tilde{U})$, renormalized $\left(U_{R}\right)$, and bare $(U)$ interaction energy parameters for three numbers of wells $N_{c}$. The interaction energy parameters are given in units of $\hbar \omega_{r}$.

\begin{tabular}{ccccc}
\hline \hline$N_{c}$ & $\alpha$ & $\tilde{U}$ & $U_{R}$ & $U$ \\
\hline 2 & 0.208 & $5.33 \times 10^{-4}$ & $5.28 \times 10^{-4}$ & $6.73 \times 10^{-4}$ \\
4 & 0.214 & $1.09 \times 10^{-3}$ & $1.08 \times 10^{-3}$ & $1.38 \times 10^{-3}$ \\
8 & 0.228 & $2.25 \times 10^{-3}$ & $2.22 \times 10^{-3}$ & $2.92 \times 10^{-3}$ \\
\hline \hline
\end{tabular}



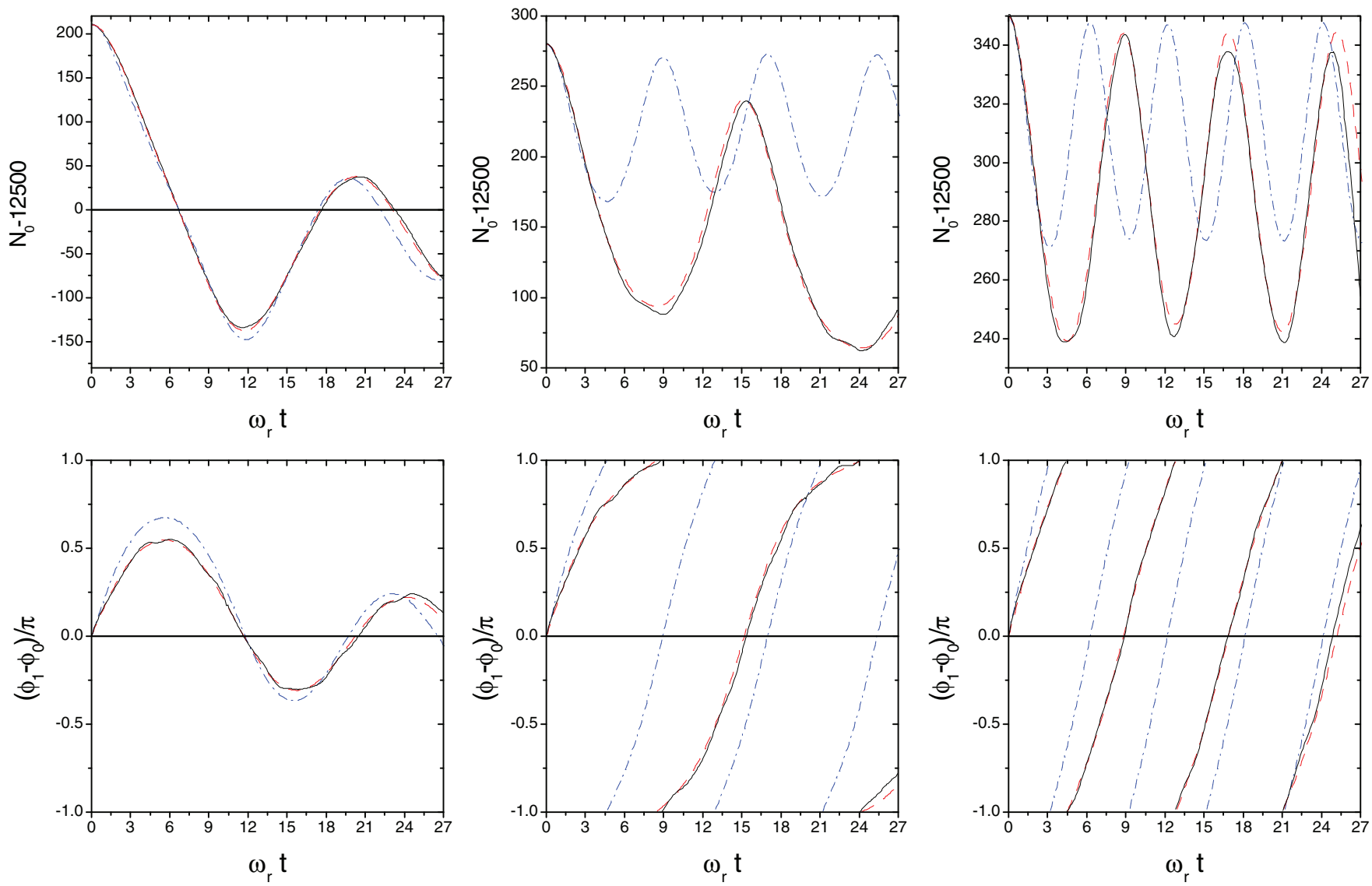

FIG. 11. (Color online) (top) Population of the $k=0$ well and (bottom) phase difference between the $k=1$ and $k=0$ wells for $N_{c}=8$ and the initial conditions $N_{0}-M=210, N_{k}-M=-30$ ( $1 \leqslant k \leqslant 7$ ) (left panels), $N_{0}-M=280, N_{k}-M=-40$ ( $1 \leqslant k \leqslant 7$ ) (middle panels), and $N_{0}-M=350, N_{k}-M=-50(1 \leqslant k \leqslant 7)$ (right panels). The solid line corresponds to the GP simulation, while the dot-dashed blue and dashed red lines correspond to the MM and RMM models, with on-site interaction energy parameters $U=2.918 \times 10^{-3} \hbar \omega_{r}$ and $U_{R}=2.2 \times 10^{-3} \hbar \omega_{r}$, respectively.

which yields

$$
\begin{aligned}
& \frac{U_{k-1}}{U} N_{k-1}-\frac{U_{k}}{U} N_{k} \\
& =(1-\alpha)\left(N_{k-1}-N_{k}\right) \\
& \quad-\alpha\left(N_{k-1}-N_{k}\right)\left[\frac{N_{c}\left(N_{k-1}+N_{k}\right)}{N}-2\right] .
\end{aligned}
$$

Finally, we replace the last result in the first term on the righthand side of Eqs. (19) and (35). By analyzing the term

$$
\begin{aligned}
& \alpha\left(N_{k-1}-N_{k}\right)\left[\frac{N_{c}\left(N_{k-1}+N_{k}\right)}{N}-2\right] \\
& \quad=\alpha\left(N_{k-1}-N_{k}\right) \frac{N_{c}\left(\Delta N_{k-1}+\Delta N_{k}\right)}{N},
\end{aligned}
$$

we first note that in the double-well case it is identically zero, while for other studied cases $\frac{N_{c}\left(\Delta N_{k-1}+\Delta N_{k}\right)}{N} \ll 1$ (cf. the range of $N_{c} \Delta N_{k} / N$ in Fig. 12). Thus, disregarding such a term, we in fact obtain a correction that can be regarded as a reduced effective interaction parameter $\tilde{U}=(1-\alpha) U$. Note that this result is in accordance with our previous analysis, which yielded the renormalized parameter $U_{R}$ using characteristic times, while the corresponding quantitative agreement is shown in Table I.

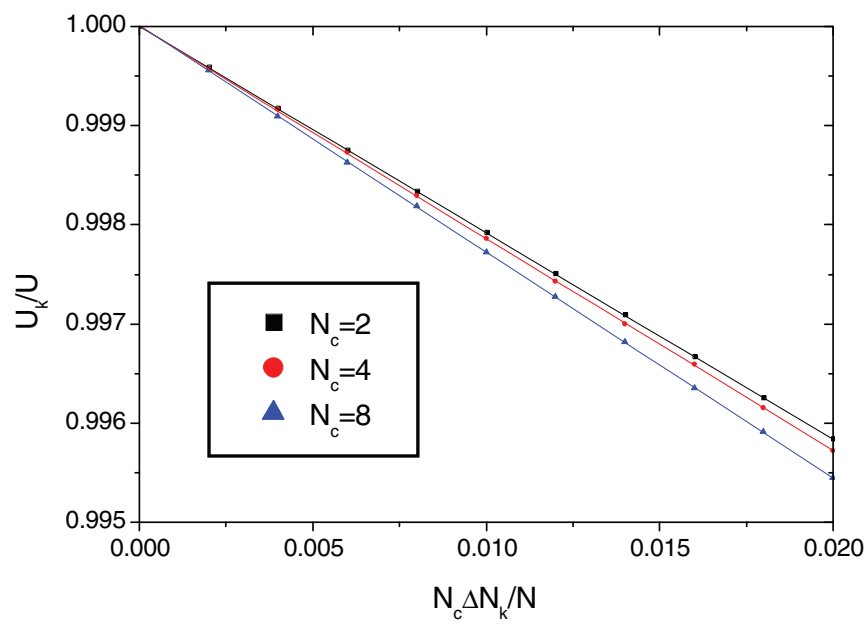

FIG. 12. (Color online) Numerical calculation of the right-hand side of Eq. (40) vs $N_{c} \Delta N_{k} / N$ for three numbers of lattice sites. The black squares, red circles, and blue triangles correspond to $N_{c}=2$, 4 , and 8 , respectively, while each line corresponds to a linear fit. 


\section{SUMMARY AND CONCLUDING REMARKS}

We have investigated the dynamics of ring-shaped optical lattices with a high number of particles per site. To this aim, we have derived the equations of motion for population and phase differences between neighboring sites of a generalized multimode model that utilizes a localized on-site Wannierlike basis. We have shown that in the case of a double-well system, this approach coincides with a recent improved twomode model [3].

To test the quality of our model, we have numerically solved the time-dependent GP equation for different numbers of wells, particularly 2, 4, and 8 . By realizing that the self-trapping time period turns out to be chiefly ruled by the on-site interaction energy parameter and utilizing the output of a single GP simulation, we were able to renormalize such a parameter. The use of this renormalized parameter in the multimode equations strikingly led to a much better agreement with the GP results for all investigated initial conditions, of which only a few representatives were included in this report. Finally, we have shown that the effective interaction energy parameter, which takes into account the deformation of the density during the time evolution, yields results that are in good agreement with those previously obtained for the renormalized parameter.

To conclude, we wish to emphasize that the two-mode model has predicted, even in its improved version [3], a sizable faster evolution than our GP simulation results, as discussed in Sec. IV C. The same behavior is observed in previous experimental and theoretical works dealing with other types of double-well systems (see, e.g., [6,23] and references therein). We believe that also in these systems as in our case, the TM model with an effective reduction of the on-site interaction energy parameter numerically calculated as proposed here should provide a more accurate dynamics.

\section{ACKNOWLEDGMENTS}

We acknowledge M. Guilleumas for a careful reading of the manuscript. D.M.J. and H.M.C. acknowledge CONICET for financial support under PIP Grants No. 11420090100243 and No. 11420100100083, respectively.
[1] A. Smerzi, S. Fantoni, S. Giovanazzi, and S. R. Shenoy, Phys. Rev. Lett. 79, 4950 (1997).

[2] S. Raghavan, A. Smerzi, S. Fantoni, and S. R. Shenoy, Phys. Rev. A 59, 620 (1999).

[3] D. Ananikian and T. Bergeman, Phys. Rev. A 73, 013604 (2006).

[4] X. Y. Jia, W. D. Li, and J. Q. Liang, Phys. Rev. A 78, 023613 (2008).

[5] M. Albiez, R. Gati, J. Fölling, S. Hunsmann, M. Cristiani, and M. K. Oberthaler, Phys. Rev. Lett. 95, 010402 (2005).

[6] M. Melé-Messeguer, B. Juliá-Díaz, M. Guilleumas, A. Polls, and A. Sanpera, New J. Phys. 13, 033012 (2011).

[7] M. Abad, M. Guilleumas, R. Mayol, M. Pi, and D. M. Jezek, Europhys. Lett. 94, 10004 (2011).

[8] T. Mayteevarunyoo, B. A. Malomed, and G. Dong, Phys. Rev. A 78, 053601 (2008); B. Xiong, J. Gong, H. Pu, W. Bao, and B. Li, ibid. 79, 013626 (2009); Q. Zhou, J. V. Porto, and S. Das Sarma, ibid. 84, 031607 (2011); B. Cui, L. C. Wang, and X. X. Yi, ibid. 82, 062105 (2010); M. Abad, M. Guilleumas, R. Mayol, M. Pi, and D. M. Jezek, ibid. 84, 035601 (2011).

[9] D. M. Jezek, P. Capuzzi, and H. M. Cataldo, Phys. Rev. A 87, 053625 (2013).

[10] C. E. Creffield, Phys. Rev. A 75, 031607(R) (2007); J.-K. Xue, A.-X. Zhang, and J. Liu, ibid. 77, 013602 (2008); T. J. Alexander,
E. A. Ostrovskaya, and Y. S. Kivshar, Phys. Rev. Lett. 96, 040401 (2006); B. Liu, L.-B. Fu, S.-P. Yang, and J. Liu, Phys. Rev. A 75, 033601 (2007).

[11] A. R. Kolovsky, Phys. Rev. A 82, 011601(R) (2010); S. K. Adhikari, J. Phys. B 44, 075301 (2011).

[12] T. F. Viscondi and K. Furuya, J. Phys. A 44, 175301 (2011).

[13] S. De Liberato and C. J. Foot, Phys. Rev. A 73, 035602 (2006).

[14] H. M. Cataldo and D. M. Jezek, Phys. Rev. A 84, 013602 (2011).

[15] A. Smerzi and A. Trombettoni, Phys. Rev. A 68, 023613 (2003).

[16] C. Ryu, M. F. Andersen, P. Cladé, V. Natarajan, K. Helmerson, and W. D. Phillips, Phys. Rev. Lett. 99, 260401 (2007).

[17] C. N. Weiler, T. W. Neely, D. R. Scherer, A. S. Bradley, M. J. Davis, and B. P. Anderson, Nature (London) 455, 948 (2008).

[18] E. P. Gross, Nuovo Cimento 20, 454 (1961); L. P. Pitaevskii, Zh. Eksp. Teor. Fiz. 40, 646 (1961) [Sov. Phys. JETP 13, 451 (1961)].

[19] Y. Castin and R. Dum, Eur. Phys. J. D 7, 399 (1999).

[20] D. M. Jezek and H. M. Cataldo, Phys. Rev. A 83, 013629 (2011).

[21] D. M. Jezek, P. Capuzzi, and H. M. Cataldo, J. Phys. B 41, 045304 (2008).

[22] K. Henderson, C. Ryu, C. MacCormick, and M. G. Boshier, New J. Phys. 11, 043030 (2009).

[23] R. Gati and M. K. Oberthaler, J. Phys. B 40, R61 (2007). 